\title{
Évolution thermique des séries sédimentaires de la Marge ardéchoise : étude pétrographique de la matière organique et des argiles
}

\author{
Luis Martinez $^{\mathrm{a}, *}$, Joëlle Duplay ${ }^{\mathrm{b}}$, Isabel Suarez-Ruiz ${ }^{\mathrm{c}}$, Jean Robert Disnar ${ }^{\mathrm{d}}$, Geneviève Farjanel ${ }^{\mathrm{e}}$, \\ Philippe Larqué ${ }^{\mathrm{b}}$, Nicole Liewig ${ }^{\mathrm{b}}$ \\ a G2R-CREGU, UMR 7566, UHP, bd des Aiguillettes, 54500 Vandoeuvre-lès-Nancy, France \\ b CGS, EOST, 1, rue Blessig, 67084 Strasbourg cedex, France \\ c INCAR, CSIC, La corredoria s/n, Apartado 73, 33080 Oviedo, Espagne \\ d ISTO, bât. Géosciences, université d'Orléans, BP 6759, 45067 Orléans cedex 2, France \\ e BRGM, av. de Concyr, BP 6009, 45060 Orléans, France
}

Reçu le 18 février 2002 ; accepté le 14 octobre 2002

Présenté par Georges Pédro

\begin{abstract}
Thermal evolution of the sedimentary series of the Ardèche Margin: petrological studies of organic matter and clays. Two wells, Balazuc (BA1) and Morte-Mérie (MM1), located in a confined area (1200 m apart) and separated by the Uzer fault (a Liassic structure with a dip fault of $1300 \mathrm{~m}$ ) were analysed using conventional methodologies and techniques (PRV, TAI, XRD, STEM) in order to compare the diagenetic evolution of clays and organic matter. The thermal convective process allows the circulation of hot fluids and the oxidation of organic matter. The conductive process allows the maturation of the organic matter, the expulsion of hydrocarbons and the deposit of pyrobitumes in the migration channels. To cite this article: L. Martinez et al., C. R. Geoscience 334 (2002) 1021-1028.
\end{abstract}

๑ 2002 Académie des sciences / Éditions scientifiques et médicales Elsevier SAS

organic matter / clays / diagenesis / Ardèche Margin / France

Résumé - Deux forages, de part et d'autre de la faille d'Uzer, marquée par un rejet de $1300 \mathrm{~m}$, sont situés à $1200 \mathrm{~m}$ de distance. Ces forages, Balazuc (BA1) et Morte-Mérie (MM1), permettent de comparer les mêmes formations ayant subi des évolutions diagénétiques différentes. Dans ces formations, l'étude de la matière organique (PRV, IAT) et des argiles (DRX, STEM) permet d'observer les variations diagénétiques produites par les variations de température et de circulation des fluides suivant la profondeur de la faille. Cette étude montre que le régime thermique convectif permet une circulation de fluides chauds (évolution contrastée des interstratifiés illite/smectite) et une oxydation de la matière organique. Le régime conductif permet, en revanche, une évolution des interstratifiés illite/smectite, la maturation de la matière organique, une expulsion des hydrocarbures et un dépôt de pyrobitumes dans les chemins de migration. Par ailleurs, les argiles et en particulier les interstratifiés illite/smectite ont une évolution contrastée, car l'évolution générale progressive liée à la profondeur est perturbée par des effets ponctuels liés aux failles (Trias-Carbonifère) ou à des environnements spécifiques (formations évaporitiques du Carnien, diagenèse organique). Pour citer cet article: L. Martinez et al., C. R. Geoscience 334 (2002) 1021-1028.

() 2002 Académie des sciences / Éditions scientifiques et médicales Elsevier SAS

matière organique / argiles / diagenèse / Marge ardéchoise / France

\section{Abridged version}

The mineralogical study of the Morte-Mérie (MM1) sediments cored between 13 and $976 \mathrm{~m}$ depth shows a monotonous succession of argillaceous sandstones to sandy limestones. These lithologic variations are underlined by the relative variations of the major mineralogical components (whole-rock XRD): quartz, feldspars, clays,

\footnotetext{
* Correspondance et tirés à part

Adresse e-mail : Luis.Martinez@g2r.uhp-nancy.fr (L. Martinez).
} 
calcite, dolomite (Fig. 2). A small faulted level occurring at $352 \mathrm{~m}$ is characterised by the presence of magnesite and by an important evaporitic level with gypsum and anhydrite that extends from the Upper Carboniferous until the Middle Carnian (between 300 and $500 \mathrm{~m}$ depth). In the clay paragenesis, illite is the dominant mineral (Fig. 2). In Balazuc, it is associated with illite/smectite mixed layers, chlorite and kaolinite on the top of the core. At $1000 \mathrm{~m}$ depth, illite becomes the remaining clay until the Carboniferous, where it is associated with chlorite. The illite crystallinity index decreases gradually with increasing depth. There is a peculiar level to notice in BA1 as well as in MM1, at the base of the Carnian, which is characterised by evaporitic minerals and where the illite is associated with corrensite. In MM1, some illite/smectite mixed-layers with low smectite content are associated to illite in several levels near fault zones. As in BA1, the IC decreases gradually with increasing depth, although great variations occur, which are probably related to the presence of faults and fluids circulations along them. The ICs are lower overall in MM1 than in BA1, but decrease more rapidly. Particle compositions of illites and I/S obtained by microprobe analyses (STEM) show the same trends with depth in MM1 and BA1, i.e., a decrease in $\mathrm{Si}, \mathrm{Fe}$ and $\mathrm{Mg}$ and increase in $\mathrm{K}$ and $\mathrm{Al}$. Great variations in $\mathrm{K}, \mathrm{Fe}$ and $\mathrm{Mg}$ are noticed in fault zones probably related to fluids circulations.

Organic matter is more abundant in MM1 than in BA1 sediments and, in general, the Upper Palaeozoic presents the richest levels. Data from Rock-Eval pyrolysis from both series reveal a mature to over-mature character of the organic matter [6-8, 14]. Therefore, catagenesis/metagenesis transition can be placed between 300
$400 \mathrm{~m}$ (Carnian/Norian limit) in MM1 and at about $1500 \mathrm{~m}$ depth in BA1 series (Fig. 4). The $T_{\max }$ values found for the Triassic sediments of MM1 are lower than $450{ }^{\circ} \mathrm{C}$, slightly increasing with increasing depth. Different results were obtained in the upper part of the BA1 series, its organic matter showing a slow degree of maturity. Consequently, both series have had a maximum effect of temperature during different times. For the Palaeozoic sediments, the $T_{\max }$ values obtained show a higher degree of maturity than that found in normal conditions of subsidence [8]. The thermal evolution of both series was confirmed by the measurements of vitrinite reflectance (PRV) (Fig. 4). This information also confirms the rise in the degree of evolution of the clay fraction during the burial of sediments. However, when the different evolution parameters are compared, it is difficult to obtain a parallelism between clay minerals and organic matter evolution, because a fraction of organic matter in BA1 well was oxidised by fluids and thermal organic evolution was retarded with a convective thermal regime (Fig. 4). In contrast, the Morte-Mérie well shows a purely conductive temperature process. This interpretation is consistent with the estimates of an erosion cover of $2 \mathrm{~km}$ over the two sites [5, 14] associated with a regional geothermal flow of $80 \mathrm{~mW} \mathrm{~m}^{-2}$ [10]. The action of fluids in clay evolution is different in those series. Moreover, clay minerals, especially illite and illite/smectite components, show a chaotic evolution due to faulted levels (Triassic/Carboniferous) and also to levels which have undergone special chemical processes (evaporitic formation of Carnian age). Finally, it has been estimated that the beginning of the gas window corresponds to the zone of the series in which the crystallinity index and the proportion of the illite/smectite mixed layers are the lowest.

\section{Introduction}

Les minéraux argileux, tout comme les macéraux organiques, sont des objets sensibles aux contraintes thermiques et mécaniques, et peuvent être utilisés comme marqueurs de l'évolution diagénétique. Mais ces contraintes diagénétiques ne s'expriment pas de façon simple. En effet, elles n'impliquent pas seulement la température, mais aussi le temps et la circulation des fluides. De plus, d'un point de vue chronologique, elles sont plus ou moins intenses au cours d'événements géologiques spécifiques, comme l'érosion ou les failles. D'une part, les marqueurs organiques enregistrent l'histoire thermique diagénétique suivant une cinétique chimique et thermique irréversible, avec formation d'hydrocarbures qui peuvent ou non migrer, suivant la circulation des fluides, avec ou sans compaction. D'autre part, la diagenèse des argiles et, en particulier, l'évolution thermique de l'indice de cristallinité de l'illite peut être réversible et très sensible aussi à la compaction et aux change- ments thermiques et chimiques de ces circulations de fluides. Dans ce travail, nous comparons l'évolution de la matière organique et celle des argiles en fonction de la profondeur d'enfouissement actuel et du contexte structural. Certaines observations pétrographiques nous font penser que les diagenèses organiques et minérales sont non seulement contemporaines, mais qu'elles enregistrent les variations thermiques de la circulation des fluides. De plus, la simple observation pétrographique indique que les interactions entre les argiles et la matière organique sont possibles dans ce contexte (Fig. 1, photo A).

\section{Contexte géologique}

Deux forages, distants de $1200 \mathrm{~m}$, ont été implantés en Ardèche en 1990 et 1993 dans le cadre du programme «Géologie profonde de la France»(GPF). Ils sont situés de part et d'autre de la faille d'Uzer, très active au Lias et dont le rejet est de 1300 m. Ces 


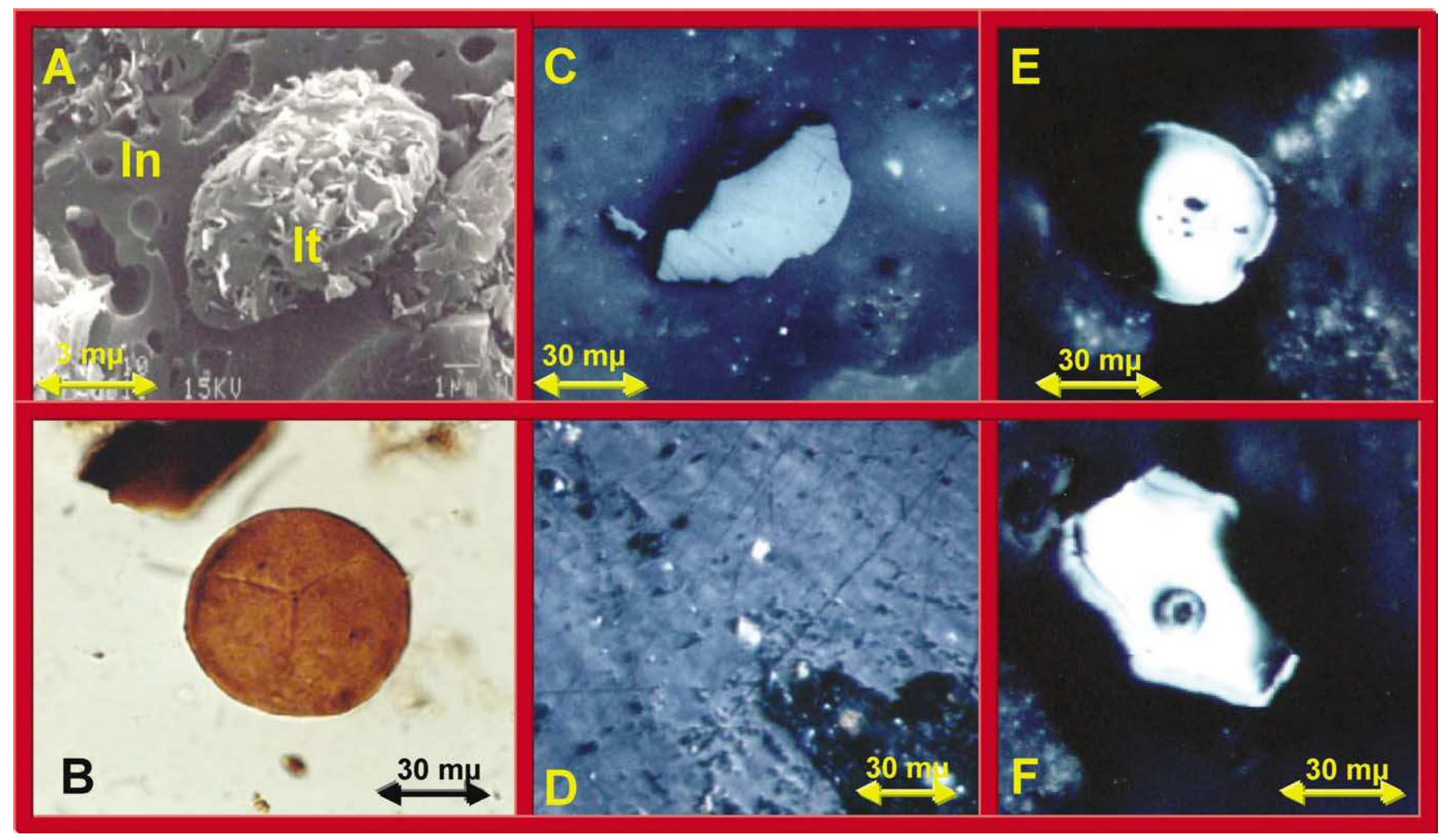

Fig. 1. Évolution thermique de la matière organique. A : Interaction de l'illite (It) avec l'inertinite dans la fenêtre huile-gaz; $\mathbf{B}:$ spore trilète dans la fenêtre huile-gaz; $\mathbf{C}$ : vitrinite dans la fenêtre à huile; $\mathbf{D}$ : vitrinite anisotrope ; $\mathbf{E}$ : pyrobitume avec une vacuole de dévolatilisation du gaz; $\mathbf{F}$ : pyrobitume avec une vacuole de dévolatilisation du gaz oxydé.

Fig. 1. Thermal evolution of organic matter. A: Illite (It)-inertinite (In) in the window gas-oil; B: threefold spore in the window gas-oil; C: vitrinite in the window gas-oil; D: anisotropic vitrinite; E: pyrobitumen with a vacuole due to gas formation; $\mathbf{F}$ : oxidation of pyrobitumen after gas formation.

forages permettent de comparer des formations semblables ayant subi des évolutions diagénétiques différentes. Les séries considérées sont celles du Lias inférieur et du Trias, qui ont pu être carottées dans les deux sites (Fig. 2). Le forage de Balazuc (BA1, $1725 \mathrm{~m}$ de profondeur) traverse le bloc effondré [9, 17]. Le forage de Morte-Mérie (MM1, $980 \mathrm{~m}$ de profondeur) traverse les formations les moins subsidentes du système [18].

\section{Méthodes d'étude}

L'étude des deux forages a été réalisée par diverses approches : pétrographique et minéralogique. La matière organique (MO) a été étudiée en microscopie optique par le pouvoir réflecteur de la vitrinite (PRV). Les caractéristiques techniques de cette méthode ont été publiées par plusieurs auteurs $[2,3,16]$. Nous avons effectué les observations en réflectance et en fluorescence optique [2]. Les mesures de PRV de MM1 ont été complétées par des études de palynofaciès pour déterminer le degré de préservation de la matière organique (indice d'altération thermique/IAT) et le type de matière organique présente dans l'en- semble des deux colonnes sédimentaires. Nous avons utilisé les résultats de géochimie organique de la pyrolyse Rock-Eval $[6,15]$ provenant des échantillons répartis sur la même colonne stratigraphique. Ils ont été pris en compte dans l'interprétation de nos résultats $\left(T_{\max }, \% \mathrm{COT}\right)$. Ils ont permis d'estimer le degré d'évolution thermique de cette matière organique et de confirmer les valeurs de PRV et d'IAT. Les argiles ont été étudiées par le STEM et par diffraction des rayons $\mathrm{X}$ suivant l'indice de cristallinité de l'illite [4].

\section{Résultats}

\section{1. Étude de la matière minérale}

La série sédimentaire forée de Morte-Mérie (MM1), entre 13 et $976 \mathrm{~m}$, est constituée par une succession assez monotone de niveaux argilo-gréseux à grésocarbonatés [18]. Les variations lithologiques se traduisent, dans la composition minéralogique, par des variations relatives des composants majeurs : quartz, feldspaths, argiles, calcite, dolomite (Fig. 2). Un petit niveau faillé à $352 \mathrm{~m}$ se différencie par la présence de magnésite. D'importants niveaux évaporitiques à gypse et anhydrite s'étendent depuis la fin du Carbo- 


\section{Forages Balazuc et Morte-Mérie}

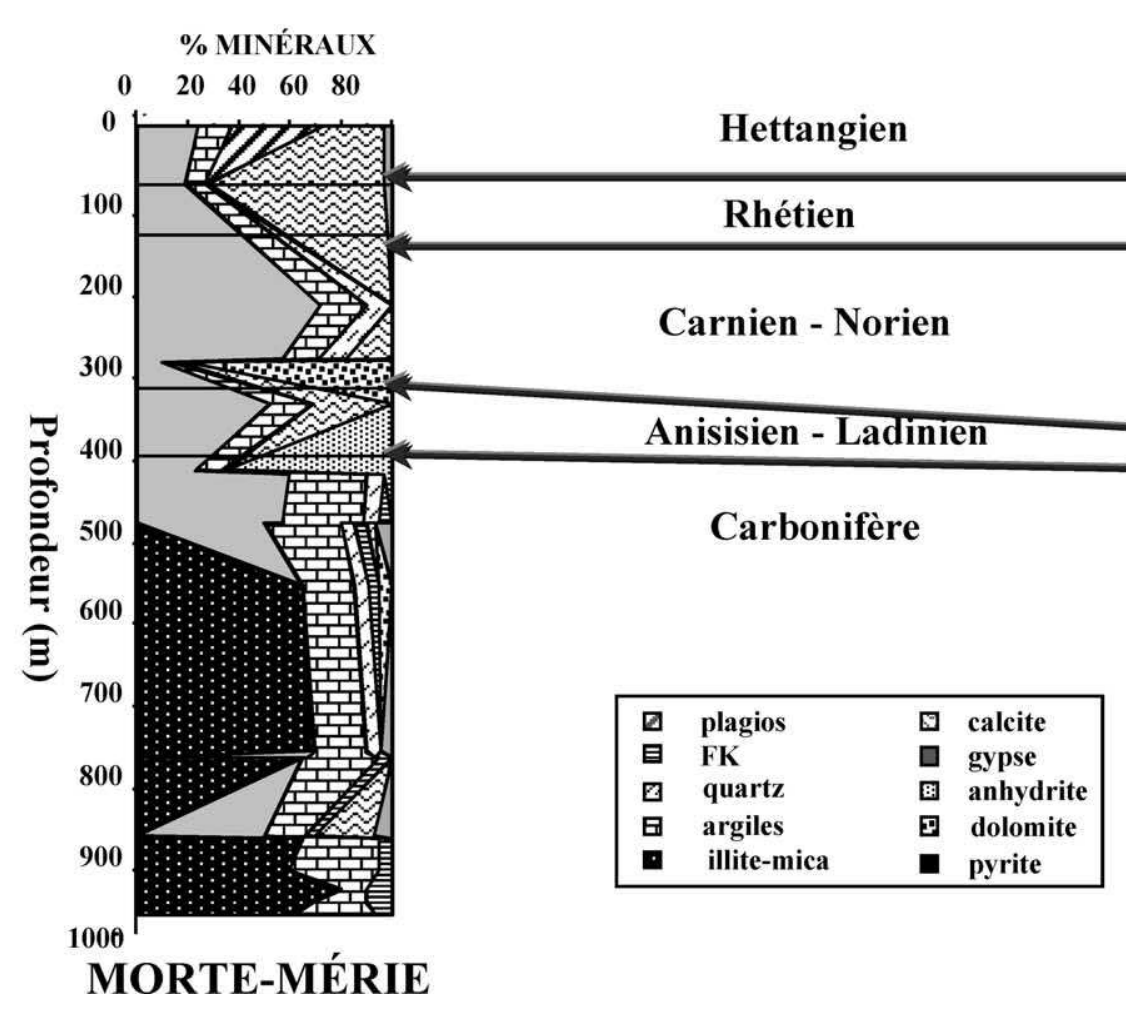

Fig. 2. Composition minérale des forages de Balazuc et de Morte-Mérie.

Fig. 2. Mineral composition of boreholes Balazuc and Morte-Mérie.

nifère jusqu'à la première moitié du Carnien (entre 300 et $500 \mathrm{~m}$ de profondeur). Les niveaux comparables de Balazuc (BA1) (de 1300 m pour le Rhétien à $1750 \mathrm{~m}$ pour le Carbonifère supérieur) montrent des spécificités minéralogiques identiques.

En ce qui concerne les paragenèses argileuses, on observe à Balazuc une association d'illite, interstratifiés illite/smectite, kaolinite et chlorite au sommet, avec diminution progressive de l'illite/smectite, de la chlorite et de la kaolinite, jusqu'à disparition totale en profondeur. À partir de $1000 \mathrm{~m}$, l'illite est la seule phase présente jusqu'au Carbonifère, mis à part les niveaux évaporitiques à anhydrite de la base du Carnien; dans ces niveaux, de la corrensite est associée à l'illite. L'indice de cristallinité de l'illite (IC) diminue du sommet vers la base du sondage, et de façon rapide entre 500 et $800 \mathrm{~m}(\mathrm{IC}=200$, puis 75$)$. Puis il évolue peu entre 800 et $1200 \mathrm{~m}(\mathrm{IC}=80)$, pour diminuer ensuite régulièrement $[4,15]$. À Morte-Mérie, l'illite est la phase argileuse dominante; elle est fréquemment associée à de l'illite/smectite tout au long du sondage, et à de la chlorite dans le Carbonifère (Tableau 1). On retrouve comme dans $\mathrm{BA} 1$, la paragenèse particulière à illite et corrensite des niveaux évaporitiques du Carnien (Tableau 1) à laquelle s'ajoute l'illite/smectite et parfois de la chlorite et de la chlorite/smectite. L'indice de cristallinité de l'illite diminue régulièrement de 125 à 60 lorsque la profondeur augmente (Fig. 3). Plus bas, les valeurs restent du même ordre (IC = 80), mais avec des fluctuations apparemment contrôlées par la présence des failles [15]. En comparant les niveaux équivalents de MM1 et BA1, on constate que la cristallinité de l'illite augmente régulièrement avec la profondeur dans les deux sondages, mais que d'importantes fluctuations sont mises en évidence [4], qui sont liées aux zones de faille. Par ailleurs, on note la présence fréquente d'illite/smectite dans ces zones, alors que seule l'illite est présente dans les niveaux équivalents de BA1. On remarque également que la cristallinité est moins bonne à MM1 qu'à BA1, mais qu'elle augmente plus rapidement. La composition de particules d'illites et I/S obtenue par microsonde électronique (STEM) a permis de suivre leur évolution chimique avec la profondeur. Globalement, on note en particulier une diminution du silicium, du fer et du magnésium, et une augmentation du potassium et de 


\section{Thermal evolution of Clay minerals in Balazuc and Morte Mérie series}

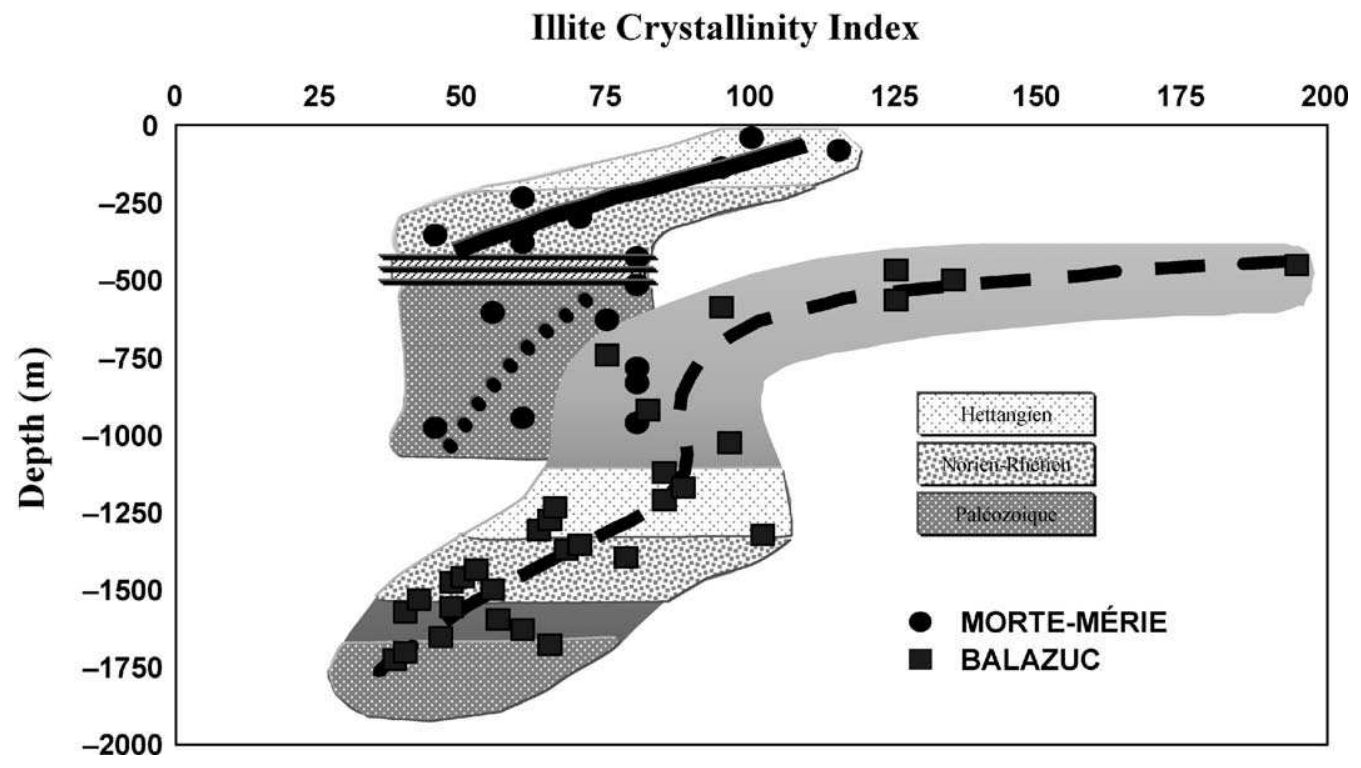

Fig. 3. Évolution thermique de l'indice de cristallinité de l'illite.

Fig. 3. Thermal evolution of the illite crystallinity index.

l'aluminium. Cependant, cette évolution est plus rapide à MM1 qu'à BA1. Par ailleurs, on constate de fortes variations en potassium, fer et magnésium dans les zones de failles, ce qui peut se traduire par des changements de conditions chimiques [4], liés aux passages de fluides hydrothermaux.

\section{2. Étude de la matière organique}

Il est bien connu qu'au cours de l'enfouissement des sédiments, la matière organique (MO) évolue thermiquement. La pyrolyse Rock-Eval a permis d'étudier les trois stades successifs de la diagenèse qui avaient déjà été identifiés dans les deux forages $[6-8,14]$ : la diagenèse, la catagenèse et la métagenèse (Fig. 4). D'une manière générale, les teneurs en MO (\%COT) de MM1 sont supérieures à celles de BA1 [14]. Les observations faites dans la partie supérieure des deux forages révèlent des degrés de maturité différents, mais un paléorecouvrement maximal d'épaisseur très voisine de $2 \mathrm{~km}$, dans les deux sites [14]. Pour le Paléozoïque, les valeurs de $T_{\max }$ [15] et de PRV (Fig. 4) indiquent un degré de maturité très élevé [7], supérieur à celui auquel la phase de subsidence peut amener le matériel considéré [8].

\section{3. Étude de pétrographie organique}

À MM1, les données de PRV $(0,73)$ ont révélé le caractère mature de la $\mathrm{MO}$, la fenêtre à huile se situe dans le Carnien/Norien à environ 300 m (Fig. 1, photo A, Fig. 2). Il est difficile de situer le début de la fenêtre à gaz (transition catagenèse/métagenèse) avec précision dans les deux sondages, car la MO a plusieurs origines [13]. En effet, elle peut être d'origine continentale, marine, voire lacustre (dans le Carnien); en revanche, dans le Paléozoïque, elle est essentiellement continentale. Dans cet article, nous avons utilisé le PRV moyen pour fixer les limites catagenèse/métagenèse. C'est dans le Paléozoïque supérieur que l'on trouve les niveaux les plus riches en MO. À MM1 comme à BA1 (près de la faille), ce niveau contient une MO riche en collinite. Dans le forage MM1, nous pouvons situer facilement le début des sédiments du Paléozoïque dans la fenêtre à huile (PRV entre 0,8 et $0,9 \%$ à $400 \mathrm{~m}$ de profondeur et un $T_{\max }$ de $440{ }^{\circ} \mathrm{C}$ ). En revanche, en BA1, la collinite est bien dans la fenêtre à gaz (PRV entre 1,9 et $2 \%$, $T_{\max }>550{ }^{\circ} \mathrm{C}$ ). La composition de la MO déduite de l'analyse optique des préparations palynologiques nous a permis d'identifier plus facilement le type de MO dans les échantillons étudiés (Fig. 1, photo B). Pour BA1, on constate que la MO amorphe disparaît à $564 \mathrm{~m}$ (Domérien) et réapparaît à $1319 \mathrm{~m}$ de profondeur (Rhétien), que le nombre de particules charbonneuses opaques augmente dans l'Hettangien, et que l'IAT augmente avec la profondeur (jusqu'à la fin de l'Hettangien, IAT $=2,5-3$ ) et diminue à $1367 \mathrm{~m}$ (début du Rhétien, IAT $=3$ ) pour augmenter ensuite de façon continue jusqu'à $1708 \mathrm{~m}$ (Carbonifère, IAT =4). À MM1, on peut retrouver, dans le Paléozoïque, des spores qui montrent un IAT qui oscille entre 3et $3+$. Ceci nous montre que la MO de BA1 est donc plus évoluée thermiquement. 


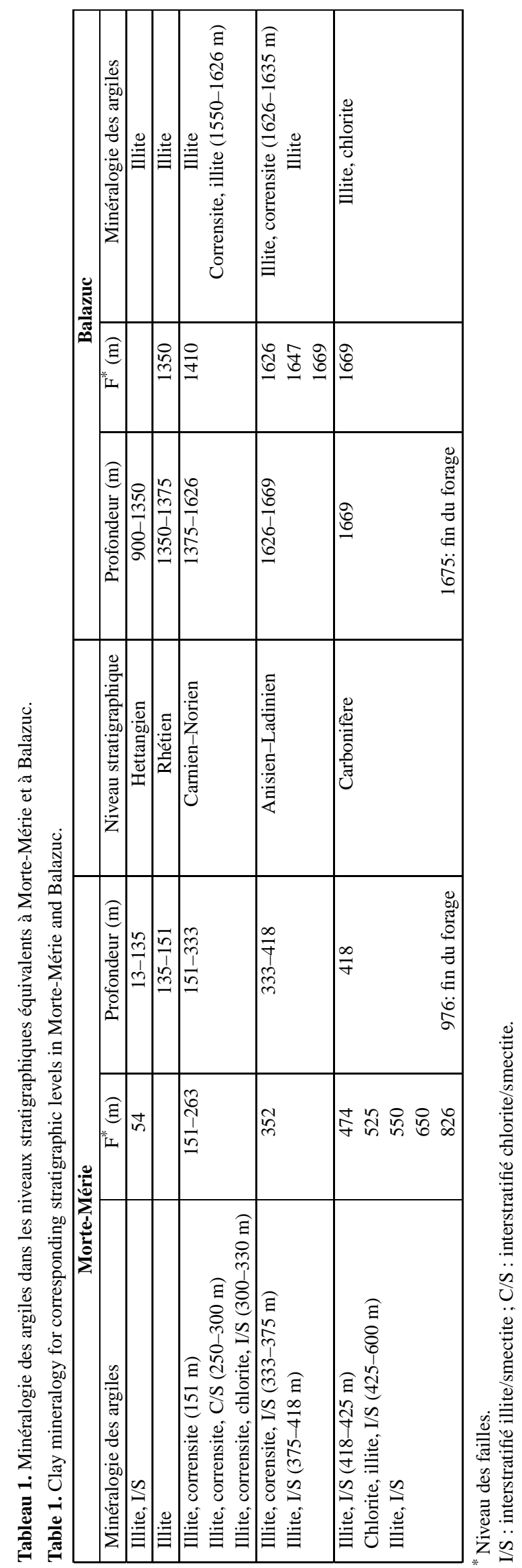

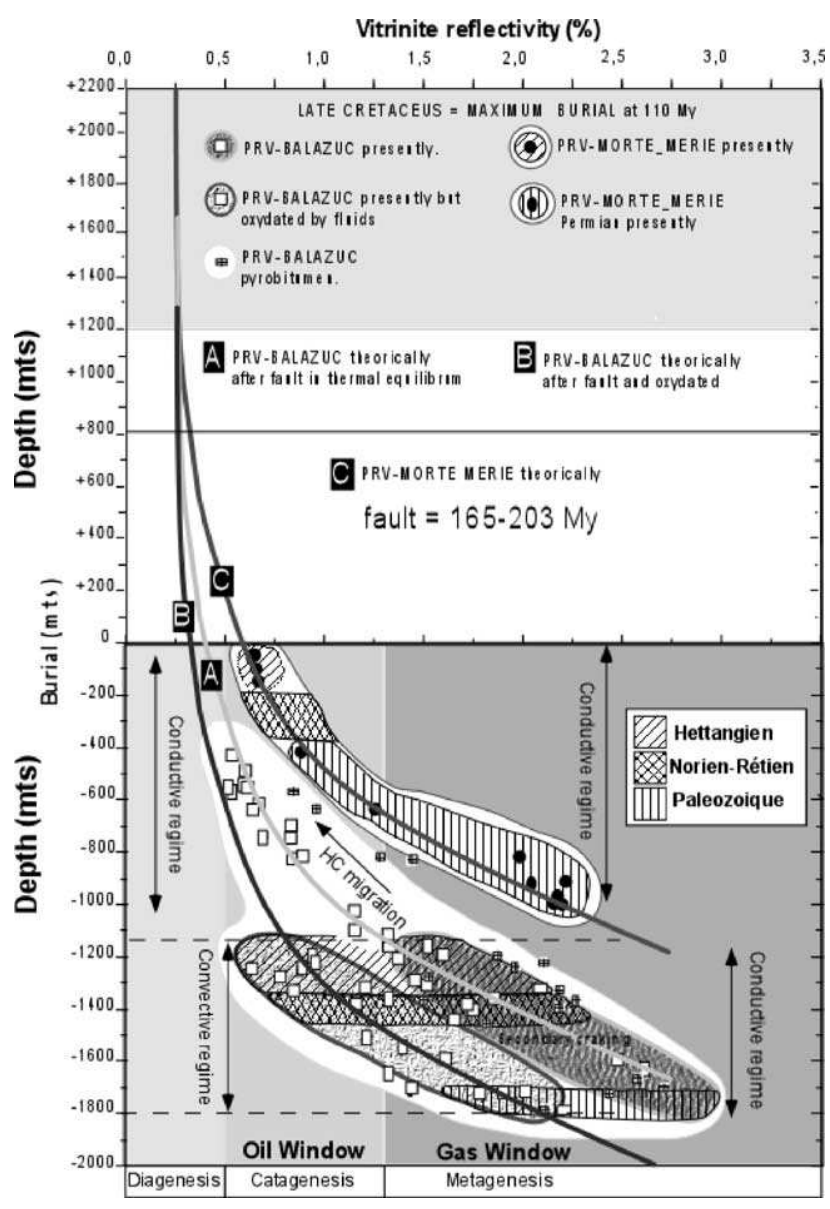

Fig. 4. Courbes théoriques de l'évolution thermique du PRV des forages de Balazuc et Morte-Mérie après le fonctionnement de la faille d'Uzer.

Fig. 4. Theoretical curves of PRV thermal evolution of Balazuc and Morte-Mérie boreholes before Uzer fault functioning.

En considérant la forme de la courbe de PRV (Fig. 4), on constate qu'à BA1, le PRV augmente de façon régulière avec la profondeur jusqu'à la fin de l'Hettangien, qu'il y a un décalage avec une diminution du PRV à $1166 \mathrm{~m}$, que cette diminution est progressive entre l'Hettangien inférieur $(1166 \mathrm{~m})$ et le Rhétien $(1367 \mathrm{~m})$ et que le PRV recommence à augmenter au niveau du Norien [16]. En revanche, à MM1, les valeurs de PRV sont moins importantes et constantes. À la limite entre l'Hettangien inférieur et le Rhétien de BA1, la vitrinite est de taille importante et devient anisotrope (Fig. 1, photo C), la MO est dans la fenêtre à gaz et le craquage secondaire est intense (Fig. 1, photo D). Au contraire, à MM1, la MO est au milieu de la fenêtre à huile et il n'y a pas de traces de craquage secondaire. En général, lorsque la matière organique rentre dans la métagenèse, une grande partie des bitumes formés dans la fenêtre à huile se transforment en gazolines et en gaz par craquage secondaire [12]. Les hydrocarbures légers migrent et le résidu solide (pyrobitumes) reste dans les 
chemins de migration [12]. Dans notre étude, les particules charbonneuses des lames palynologiques sont en grande partie des pyrobitumes souvent arrondis, de petite taille et microporeux (Fig. 1, photo E). En microscopie en réflexion, ils présentent une légère anisotropie et leur biréfringence augmente de façon non régulière avec la profondeur.

\section{Interprétation}

$\mathrm{Si}$ on considère les expériences de maturation artificielle du même type de matière organique [12], l'expulsion des bitumes peut s'observer lorsque le PRV oscille entre 1,2 et 1,3\% et que les pyrobitumes commencent à apparaître. Cela veut dire que, dans l'Hettangien, la fenêtre à gaz a probablement été atteinte et que dans ce contexte les bitumes ont pu alors migrer vers le Domérien (fenêtre à huile) et se transformer en gaz et en pyrobitumes en traversant le Norien et le Rhétien (Fig. 4).

\subsection{Interprétation du profil thermique de deux forages suivant la forme des courbes de PRV}

Il est bien connu, en particulier des pétroliers, que la forme de la courbe de PRV varie avec la profondeur et peut être interprétée en fonction de l'évolution de l'histoire thermique du bassin sédimentaire [1, 2]. En effet, la transformation thermique de la matière organique suit la loi d'Arrhenius et la réaction chimique est contrôlée essentiellement par le temps et la température (donc par l'histoire de la subsidence, de l'érosion et de la compaction). Donc la forme des courbes de PRV de BA1 et MM1 peut s'expliquer suivant la dynamique de la faille (Fig. 4). En effet, on peut constater qu'entre la surface et $600 \mathrm{~m}$ de profondeur, l'allure des deux courbes est similaire et normale. La seule différence est qu'à BA1 la courbe reste dans la fenêtre à huile et qu'à MM1 elle se déplace de la fenêtre à huile vers la fenêtre à gaz. On peut expliquer ce phénomène par le fait qu'avant la faille, cette partie de Balazuc avait une matière organique immature, car la MO était située de 1300 à 2000 m au-dessus de Morte-Mérie. En revanche, MM1 était déjà probablement dans la fenêtre à huile [15].

\subsection{Variation de la forme des courbes de PRV suivant la circulation des fluides}

Après le basculement de la faille, la distribution du flux de chaleur et les régimes thermiques de part et d'autre de la faille ont changé [10]. La matière organique de MM1 a subi une contrainte thermique sans circulation de fluides (régime conductif, Fig. 4, courbe C), probablement moins forte que celle de BA1 (régime convectif et conductif, Fig. 4, courbes
$\mathbf{A}$ et $\mathbf{B})$. En effet, lorsqu'on rajoute à la figure les valeurs de PRV du matériel organique oxydé par la circulation de fluides (Fig. 1, photo F, Fig. 4, courbe B) entre 1150 et $1700 \mathrm{~m}$ de profondeur, il apparaît, dans le nuage des points, deux courbes d'évolution de la vitrinite. Ceci peut s'expliquer par le ralentissement de l'évolution du PRV dû à l'oxydation produite par la circulation de fluides probablement chauds (entre 1150 et $1350 \mathrm{~m}$ de profondeur). Celle-ci est visible autour de certaines particules de vitrinite et de pyrobitumes (Fig. 1, photos $\mathbf{E}$ et $\mathbf{F}$ ). En effet, suivant l'importance et la chronologie de la circulation de fluides dans les strates, la matière organique qui est en contact avec le fluide est oxydée et ceci se traduit par une diminution significative de la taille et de la quantité des pyrobitumes, qui peuvent parfois être aussi oxydés (Fig. 1, photos $\mathbf{E}$ et $\mathbf{F}$ ). En revanche, la matière organique non oxydée continue son évolution thermique suivant un régime conductif similaire à celui de Morte-Mérie (l'allure de la courbe $\mathbf{C}$ est la même que celle de la courbe $\mathbf{A}$, mais à une profondeur différente). En effet, les particules de vitrinite non oxydée en BA1 dans cet intervalle de profondeur présentent une évolution thermique classique, avec une température plus forte et une production de bitumes et de pyrobitumes non oxydés, qui montrent l'importance du craquage secondaire et de la migration des hydrocarbures.

\section{Conclusions}

Les sédiments de BA1 et MM1 montrent que le degré d'évolution de la fraction argileuse et de la MO croît avec l'enfouissement. Mais la comparaison entre les différents marqueurs est délicate. D'une part, la MO présente un caractère mature à très mature, variable en fonction de ses différentes origines (lacustre, marine et continentale) et de sa position stratigraphique dans les deux sondages. D'autre part, les argiles, et en particulier les interstratifiés illite/smectite, présentent une évolution contrastée, car l'évolution générale progressive liée à la profondeur est perturbée par des effets ponctuels liés aux failles (TriasCarbonifère) ou à des environnements spécifiques (formations évaporitiques du Carnien, diagenèse organique). Néanmoins, malgré les fluctuations de détail, la maturité de la MO et l'évolution des argiles sont plus intenses à BA1 (Fig. 4, positions $\mathbf{A}$ et $\mathbf{B}$ par rapport à $\mathbf{C}$ ), ce qui est en bon accord avec le fait que BA1 est situé dans le panneau effondré en position de bassin. L'évolution plus intense de BA1 rendrait compte alors de la dynamique d'enfouissement plus rapide de ce bloc et de la variation du régime thermique qui en résulte (conductif et convectif). Dans ce schéma d'ensemble, étant donné l'intensité des transformations atteintes par les niveaux 
les moins enfouis, il est possible d'envisager que la diagenèse anté-effondrement, dont le témoin est MM1, pourrait ne pas être liée seulement à l'enfouissement précoce commun (2000 à $2200 \mathrm{~m}$ de sédiments supposés) [9], mais aussi à une diagenèse thermique contrôlée par la géodynamique globale, comme cela a déjà été suggéré dans d'autres contextes pour des périodes équivalentes [11]. En conclusion, cette étude montre que, même si le gradient géothermique n'est pas très diffèrent d'un bloc par rapport à l'autre après le basculement, la profondeur la plus importante permet une évolution diagénétique qui est contrôlée essentiellement par les variations des régimes thermiques conductif et convectif et par les réactions d'interaction qui accompagnent la circulation des fluides.

Remerciements. Cette étude a été financée par l'Institut des sciences de l'Univers (Centre national de la recherche scientifique), le Bureau de recherches géologiques et minières et le ministère de la Recherche et de la Technologie, à travers le programme national GPF. Nous remercions spécialement M. Steinberg (université Paris-Sud) et J.-F. Sureau (BRGM) pour leur soutien et leurs critiques constructives tout le long de cette étude.

\section{Références}

[1] P. Allen, N.J. Allen, Thermal history, in : Basin Analysis: Principles and Applications, Blackwell Scientific Publications, London, 1990, pp. 282-308.

[2] B. Alpern, D. Cheymol, Réflectance et fluorescence des organoclastes du Toarcien du Bassin de Paris en fonction de la profondeur et de la température, Rev. IFP 33 (4) (1978) 515-535.

[3] P. Bertrand, B. Pradier, Optical methods applied to source rock study, in: M.L. Bordenave (Ed.), Applied Petroleum Geochemistry, Technip, Paris, 1993, pp. 281-310.

[4] N. Clauer, F. Weber, F. Gauthier-Lafaye, T. Toulkeridis, J.-P. Sizun, Mineralogical, geochemical (REE) and isotopic (K-Ar, Rb$\mathrm{Sr}, \delta^{18} \mathrm{O}$ ) evolution of the clay minerals from faulted, carbonaterich, passive paleomargin of southeastern Massif Central, France, J. Sediment. Res. 67 (5) (1997) 923-934.

[5] Y. Copard, J.-R. Disnar, J.-F. Becq-Giraudon, M. Boussafir, Evidence and effects of fluid circulation on organic matter in intramontane coalfields (Massif Central, France), Int. J. Coal Geol. 44 (2000) 49-68.

[6] J.-R. Disnar, F. Marquis, S.M. De Witte, L. Martinez, Organic matter diagenesis of the Jurassic, Triassic and Carboniferous section penetrated in the Balazuc-1 GPF well (Ardèche margin, France), in : VIth Int. Symp. on Observation of the Continental Crust through Drilling, Paris, 1992, p. 87 (abstract).

[7] J.-R. Disnar, I. Barsonny, R. Burwoord, S.M. De Witte, J. Drouet, J. Espitalié, G. Farjanel, F. Marquis, L. Martinez, B. Pradier, Géochimie organique et reconstitution de l'histoire thermique et tectono-sédimentaire de la Marge ardéchoise (Programme GPF, France), Séance spécialisée de la SGF «Du sédiment au réservoir : quantification et modélisation de la diagenèse », Poitiers, France, 1995.

[8] J.-R. Disnar, F. Marquis, J. Espitalié, L. Barsonny, J. Drouet, D. Giot, Géochimie organique et reconstitution de l'histoire thermique et tectono-sédimentaire de la Marge ardéchoise (Programme GPF, France), Séance spécialisée de la $\mathrm{SGF}$ «Du sédiment au réservoir : quantification et modélisation de la diagenèse», Bull. Soc. géol. France 1 (1997) 73-81.

[9] D. Giot, F. Roure, S. Elmi, D. Lajat, M. Steinberg, Découverte d'accidents distensifs majeurs d'âge Jurassique sur la marge continentale du bassin du Sud-Est, Ardèche, France (programme GPF), C. R. Acad. Sci. Paris, Ser. II 312 (1991) 747-754.
[10] C. Le Carlier de Veslud, J.-J. Royer, B. Gérard, M. Pagel, Modélisation des transferts hydrogéologiques et thermiques lors de l'évolution de la paléo-marge ardéchoise (France), Bull. Soc. géol. France 169 (1) (1998) 81-89.

[11] N. Liewig, J.R. Mossmann, N. Clauer, Datation isotopique $\mathrm{K}-\mathrm{Ar}$ d'argiles diagénétiques de réservoirs gréseux : mise en évidence d'anomalies thermiques du Lias inférieur en Europe nordoccidentale, C. R. Acad. Sci. Paris, Ser. II 304 (1987) 707-711.

[12] L. Martinez, La migration primaire des hydrocarbures : étude intégrée de pétrographie et géochimie organique des roches mères argilo-détritiques, thèse d'État, université d'Orléans, France, 1993, p. 192.

[13] L. Martinez, I. Suarez Ruiz, J. Duplay, F. Weber, J.-R. Disnar, B. Romand, P. Larqué, G. Farjanel, N. Liewig, Contribution to the reconstitution of the thermal history of sedimentary series of the Ardèche Margin: study of clay and organic matter evolution in Balazuc and Morte-Mérie sites (France), in: J.O. Grimalt, C. Dorronsoro (Eds.), Selected papers from the 17th International Meeting on Organic Geochemistry, San Sebastian, Espagne, 1995, pp. 515516.

[14] M. Pagel, J.-J. Braun, J.-R. Disnar, L. Martinez, C. Renac, G. Vasseur, Thermal history constraints from organic matter, clay minerals, fluid inclusions, apatite fission tracks and stable isotopes studies at the Ardèche paleo-margin in the L'Argentière $\mathrm{Pb}-\mathrm{Zn}$ deposit area (GPF Programme, France), J. Sediment. Res. 67 (1) (1997) 235-245.

[15] B. Romand, Contribution à l'histoire thermique des séries sédimentaires de la marge ardéchoise (forage de Morte-Mérie, GPF4) : étude des argiles en relation avec la matière organique, par microscopie électronique à transmission (MET et STEM), Mémoire de maîtrise des sciences de la Terre, université Louis-Pasteur, Strasbourg, France, 1993.

[16] E. Stach, M.-T. Machowsky, M. Teichmuller, G.H. Taylor, D. Chandra, R. Teichmuller, Textbook of Coal Petrology, 3rd edn., Bornträger, Berlin, Stuttgart, 1982.

[17] J.-F. Sureau, M. Steinberg, Forages scientifiques de l'Ardèche : les interactions fluides-roches sur une paléomarge distensive : le forage de Balazuc-1. Synthèse des travaux à la fin 1992. Phase II : Les propositions de forage, Doc. BRGM (1992) 218-312.

[18] J.-F. Sureau, Forages scientifiques de l'Ardèche : les interactions fluides-roches sur une paléomarge distensive : forage Morte-Mérie-1, rapport d'exécution et données préliminaires, Doc. BRGM 229 (1992). 